\title{
QCM sensing of bisphenol A using molecularly imprinted hydrogel/conducting polymer matrix
}

\author{
Kazuya Matsumoto ${ }^{1}$, Brylee David B Tiu ${ }^{2}$, Akifumi Kawamura ${ }^{1,3}$, Rigoberto C Advincula ${ }^{2}$ and \\ Takashi Miyata ${ }^{1,3}$
}

Molecular imprinting is a well-known fabrication technique for designing artificial receptors and molecular sensors. The technique resembles a lock and key mechanism and utilizes shape-complementary cavities within polymeric materials as molecular recognition sites for various relevant molecules. In this study, we prepared molecularly imprinted polypeptide gel layers based on cyclodextrin-modified poly(L-lysine) (CD-PLL) on quartz crystal microbalance (QCM) sensor chips and investigated their molecular recognition behaviors for bisphenol A (BPA) using the QCM technique. With BPA as the template and CD as its ligand, the BPA-imprinted CD-PLL gel layers were prepared on electropolymerized polyterthiophene films, which were formed using electrochemical QCM (EQCM). The BPA-imprinted CD-PLL gel layer chip exhibited a much greater QCM response than the non-imprinted gel layer chip in an aqueous BPA solution. The greater response of the BPA-imprinted CD-PLL gel layer chip means that molecular imprinting enabled $C D$ ligands to be arranged at optimal positions for forming molecular recognition sites. The combination of in situ electropolymerization using EQCM and molecular imprinting provides useful methods for fabricating highly selective and sensitive sensor devices for monitoring minute amounts of BPA in water.

Polymer Journal (2016) 48, 525-532; doi:10.1038/pj.2016.23; published online 17 February 2016

\section{INTRODUCTION}

Bisphenol A (BPA), 2, 2-bis(4-hydroxyphenyl) propane, has been most commonly used as a monomer in manufacturing polycarbonate plastics and epoxy resins, which are extensively employed in various consumer goods and products. Despite its widespread use, many reports claim that BPA can leach out from plastic products such as food packaging materials and act as an endocrine disrupting chemical. Endocrine disrupting chemicals are exogenous agents that interfere with natural hormones of the body. These hormones are responsible for maintaining homeostasis and regulating developmental processes. ${ }^{1}$ High blood levels of BPA are associated with reproduction dysfunction, endometrial hyperplasia, recurrent miscarriages, abnormal karyotypes and polycystic ovarian syndrome. ${ }^{2-4}$ In general, gas chromatography and high-performance liquid chromatography have been used for the quantitative determination of BPA in environmental water and packaged food product samples. In particular, a uniquely sensitive sensor of BPA with a less invasive approach for evaluation in environmental and medical fields will be in considerable demand.

Molecularly imprinted polymers with molecular recognition abilities have been significant tools for the development of microand multi-analyte sensors. ${ }^{5-10}$ The molecular imprinting technique is an effective and robust method for obtaining recognition elements for chemical and biological sensors because it involves the formation of shape-complementary cavities that re-capture the target analyte within the polymer networks. In standard molecular imprinting, ${ }^{11-14}$ after prearrangement of ligand monomers around a template molecule (a target molecule) by their specific interaction, ligand monomers are copolymerized with a large number of cross-linkers. Then, molecularly imprinted polymers with molecular recognition sites are obtained by extracting the template molecule from the resulting networks. In molecular imprinting, various functional monomers with carboxy, amino and hydroxy groups have been used as ligand monomers that can form non-covalent hydrogen-bonding interactions with the template molecule. In addition, cyclodextrins (CDs), which have an apolar cavity and a hydrophilic exterior, have been reported to be useful ligands in designing molecularly imprinted polymers because they form inclusion complexes with a variety of guest molecules. ${ }^{15}$ For example, molecularly imprinted polymeric receptors were prepared by cross-linking $\beta-C D$ with a large amount of diisocyanate in the presence of various steroids as templates. ${ }^{16}$ The significant binding activities and high selectivities of the CD-cross-linked polymeric receptors toward target steroids revealed that the CD ligands are very effective in molecular imprinting.

\footnotetext{
${ }^{1}$ Department of Chemistry and Materials Engineering, Kansai University, Osaka, Japan; ${ }^{2}$ Department of Macromolecular Science and Engineering, Case Western Reserve University, Cleveland, OH, USA and ${ }^{3}$ Organization for Research and Development of Innovative Science and Technology, Kansai University, Osaka, Japan Correspondence: Professor RC Advincula, Department of Macromolecular Science and Engineering, Case Western Reserve University, 2100 Adelbert Road, Cleveland, $\mathrm{OH}$ 44106, USA

E-mail: rca41@case.edu

or Professor T Miyata, Department of Chemistry and Materials Engineering, Kansai University, 3-3-35, Yamate-cho, Suita, Osaka 564-8680, Japan.

E-mail: tmiyata@kansai-u.ac.jp

Received 11 December 2015; revised 18 January 2016; accepted 19 January 2016; published online 17 February 2016
} 
Stimuli-responsive hydrogels have attracted significant attention as smart soft materials because they can undergo reversible swelling/shrinking in response to external stimuli such as $\mathrm{pH}^{17,18}$ and temperature. ${ }^{19-21}$ Specifically, $\mathrm{pH}$-responsive hydrogels that change their volume due to $\mathrm{pH}$ variation can be synthesized by introducing ionizable groups, such as carboxy and amino groups, into the polymer networks. Such stimuli-responsive hydrogels have been investigated for many biomedical uses including sensor systems, drug delivery carriers and actuators. $^{22-26}$ Taking advantage of these desirable properties, we functionalized stimuli-responsive hydrogels with biomolecular recognition abilities based on a novel strategy that uses biomolecular complexes as reversible cross-links of their networks. ${ }^{27-30}$ Biomolecule-responsive hydrogels, which are designed using this strategy, change their volume in response to target biomolecules such as glucose, enzymes and antigens. Furthermore, the arrangement of ligands within hydrogel networks via molecular imprinting enabled us to prepare biomolecule-responsive hydrogels with molecular recognition sites that can shrink gradually according to the target molecule concentration. Our technique differs from the standard molecular imprinting approach. Specifically, we designed molecularly imprinted hydrogels that shrink by forming complexes between ligands and target molecules using minute amounts of crosslinkers. For example, BPA-imprinted hydrogels that undergo changes in volume in response to BPA were strategically prepared using molecular imprinting that used $\beta$-CDs as ligands for BPA and minute amounts of cross-linkers. ${ }^{31}$ BPA-imprinted hydrogels exhibited a significant shrinkage in the presence of BPA as the formation of an inclusion complex of BPA with two CDs resulted in a cross-linking density increase. Such molecularly imprinted hydrogels with molecular recognition sites can provide useful applications for molecular sensors, separation technology and drug delivery system (DDS) carriers. Recently, we designed stimuli-responsive hydrogels using polypeptides, such as poly(L-lysine) (PLL), as the main chain of a hydrogel network. We used polypeptides because of their well-defined structures and useful functional groups. ${ }^{32}$ Previous studies on stimuli-responsive polypeptide hydrogels revealed that polypeptides have high potential as main chains for designing molecularly imprinted hydrogels.

The formation of polymer films with molecular recognition sites on sensor chips is the most important step in fabricating sensors with high sensitivity and selectivity. In general, polymer films are prepared via solvent casting, polymer grafting and vapor deposition. ${ }^{33,34}$ Among these methods, electropolymerization is a unique technique for preparing thin polymer films via in situ polymerization from an electrode surface because the thickness, surface growth and morphology can be easily controlled by varying different process parameters (for example, scan rate and potential window). ${ }^{35,36}$ $\pi$-Conjugated or electrically conducting polymers, whose thin films can be easily formed using electropolymerization, have been investigated as sensors, ${ }^{37}$ electro-optical materials ${ }^{38}$ and semiconducting devices. $^{39}$ In general, electropolymerizable monomers, such as thiophene, aniline, pyrrole and carbazole, have been widely used to form $\pi$-conjugated or electrically conducting polymer films in electro-optical applications. ${ }^{40}$ For example, electropolymerization of terthiophene leads to the formation of highly electrochromic and conducting polythiophenes. ${ }^{41-43}$ The mechanism is based on a radical cation coupling that can be accessed using potentiodynamic or potentiostatic methods. Conjugated polymer films that are prepared via surface modification of conducting films with functional polymers provide several opportunities for taking advantage of the properties of both polymers.
In this study, we prepared BPA-imprinted polypeptide gel layers on a conducting polymer via chemical cross-linking of CD-PLL and investigated their molecular recognition behaviors. This paper focuses on the preparation of BPA-imprinted CD-PLL gel layers on polyterthiophene modified quartz crystal microbalance (QCM) sensor chips. Electrochemical QCM (EQCM) was used to probe in situ electropolymerization of polyterthiophene on the Au-electrode surface. The BPA-imprinted CD-PLL gel layers were strategically prepared via molecular imprinting using $\beta-C D$ as a ligand on a polyterthiophene surface that was produced using electropolymerization. The resulting polymer thin films were characterized using atomic force microscopy (AFM), Fourier transform infrared reflection absorption spectrometry (FT-IR-RAS), ellipsometry and static water contact angle measurements. The sensitivity and selectivity of BPA-imprinted CD-PLL gel layers for BPA were evaluated using the QCM technique, which has been used to detect nanomolar to micromolar masses. This paper demonstrates that the combination of QCM and molecular imprinting enables us to fabricate highly selective and sensitive sensor devices with commercialization potential.

\section{EXPERIMENTAL PROCEDURE}

\section{Materials}

PLL hydrobromide (12 $000 \mathrm{MWCO}$ ) and ethylene glycol diglycidyl ether were purchased from Peptide Ins. Co. Ltd (Osaka, Japan) and Tokyo Chemical Ind. Co. Ltd (Tokyo, Japan), respectively. All aqueous solutions were prepared with ultra-pure water (Milli-Q, $18.2 \mathrm{M} \Omega \mathrm{cm}$ ). The other solvents and reagents were of analytical grade, were obtained from commercial sources and were used without further purification.

\section{Synthesis of Mono-6-carboxy-6-deoxy- $\beta$-CD (carboxy-CD)}

Mono-6-amino-6-deoxy- $\beta$ - $\mathrm{CD}\left(\mathrm{NH}_{2}-\mathrm{CD}\right)$ was synthesized according to the previously reported literature. ${ }^{31,44} \mathrm{NH}_{2}$-CD $(4.0 \mathrm{~g}, 3.52 \mathrm{mmol})$ was dispersed in $30 \mathrm{ml}$ of ultra-pure water. Succinic anhydride $(0.352 \mathrm{~g}, 4.2 \mathrm{mmol})$ was added to the solution. The solution was stirred at $60^{\circ} \mathrm{C}$ and was allowed to react for $6 \mathrm{~h}^{45-47}$ After the prescribed time, the solution was poured into acetone $(300 \mathrm{ml})$. The supernatant was removed via filtration, and the precipitate was concentrated under the reduced pressure to yield carboxy-CD $(2.9 \mathrm{~g}, 66.4 \%$ yield).

\section{Introduction of CD to PLL}

Carboxy-CD (1.0 g, $0.81 \mathrm{mmol})$ was dissolved in $150 \mathrm{ml}$ of ultra-pure water. 1-Ethyl-3-(3-(dimethylamino)-propyl)carbodiimide (EDC; $0.77 \mathrm{~g}, 4.0 \mathrm{mmol}$ ) and $\mathrm{N}$-hydroxysuccinimide $(0.46 \mathrm{~g}, 4.0 \mathrm{mmol})$ were added to the aqueous solution with carboxy-CD and allowed to react for $1 \mathrm{~h}$ at room temperature. PLL ( $0.84 \mathrm{~g}, 4.0 \mathrm{mmol}$ primary amines) that was dissolved in $50 \mathrm{ml}$ of ultrapure water was added to the aqueous solution with an activated carboxy-CD. The reaction proceeded for $4 \mathrm{~h}$ at room temperature (Scheme 1). Then, the reaction mixture was poured into seamless cellulose tubing (molecular weight cut off: 3500) and was dialyzed against ultra-pure water for purification. The resultant CD-PLL was obtained via freeze-drying. The content of CD that was introduced to PLL was determined from the ${ }^{1} \mathrm{H}$ NMR proton integration. CD-PLL was dissolved in $\mathrm{D}_{2} \mathrm{O}$, and ${ }^{1} \mathrm{H}$ NMR was recorded on JEOL JNM-AL 400 spectrometer using tetramethylsilane as the internal standard. ${ }^{1} \mathrm{H}$ NMR measurements revealed that the average degree of $\mathrm{CD}$ substitution in the resulting CD-PLL was 0.20 (that is, on average one $\mathrm{CD}$ for every five lysine units).

\section{Preparation of poly(G03T-COOH) films}

After 2-(2, 5-di(thiophen-2-yl)thiophen-3-yl)acetic acid (G03T-COOH) was synthesized using a previously described method, ${ }^{42}$ its electropolymerization was performed on a QCM sensor chip via cyclic voltammetry (CV) in acetonitrile with $0.1 \mathrm{M}$ tetrabutylammonium hexafluorophosphate. Polyterthiophene-carboxylic acid films, poly $(\mathrm{G} 03 \mathrm{~T}-\mathrm{COOH})$, were prepared via electropolymerization of G03T-COOH using EQCM (Q-Sense E4 and E1, 


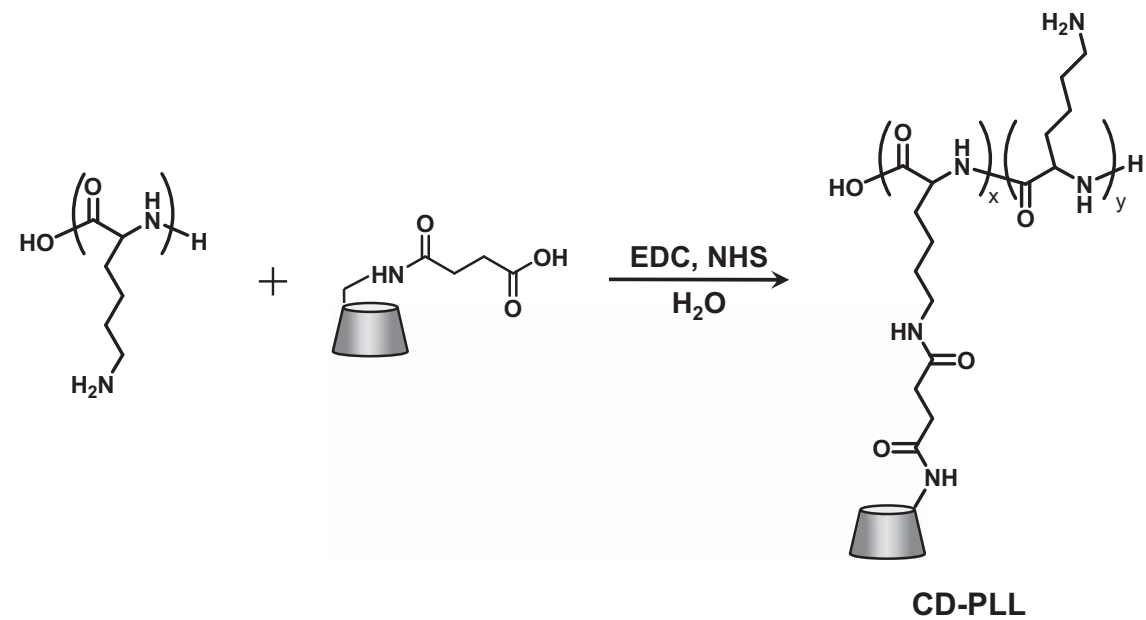

Scheme 1 Introduction of CD into PLL. A full color version of this scheme is available at Polymer Journal online.

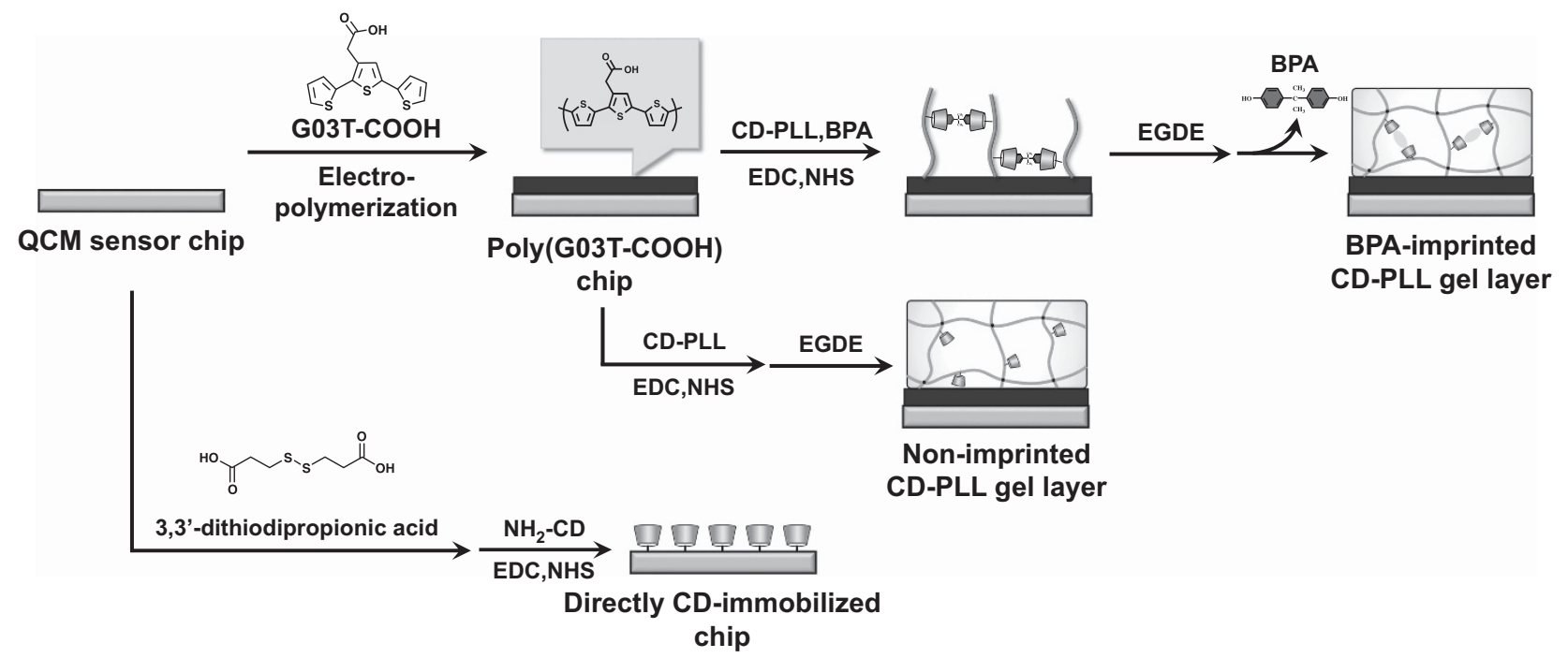

Scheme 2 Preparation of the BPA-imprinted CD-PLL gel layer chip, non-imprinted CD-PLL gel layer chip and directly CD-immobilized chip. A full color version of this scheme is available at Polymer Journal online.

Meiwa Fosis Co. Ltd, Tokyo, Japan). An AT-cut polished QCM sensor chip $(4.95 \mathrm{MHz}$ ) with a $14-\mathrm{mm}$ diameter, a $\mathrm{Ag} / \mathrm{AgCl}$ electrode (Dri-Ref, World Precision Instrument Inc., Sarasota, FL, USA) and a Pt sheet were used as a working electrode, a reference electrode and a counter electrode, respectively. QCM crystals were cleaned in a Piranha solution (a mixture of $98 \% \mathrm{H}_{2} \mathrm{SO}_{4}$ and $30 \% \mathrm{H}_{2} \mathrm{O}_{2}, 3: 1 \mathrm{v} / \mathrm{v}$ ) at $70{ }^{\circ} \mathrm{C}$ for $5 \mathrm{~min}$, rinsed with ultra-pure water and dried under nitrogen flow. The QCM sensor chips were cleaned using a ultraviolet ozone cleaner (BioForce Nanoscience, Inc., Ames, IA, USA) for $10 \mathrm{~min}$ before use. The poly(G03T-COOH) films were formed by scanning the potential between 0 and $1100 \mathrm{mV}$ at a scan rate of $100 \mathrm{mV} \mathrm{s}^{-1}$ for 40 cycles using CV techniques. Mass changes on the QCM sensor chips were monitored using EQCM to in situ evaluate oxidation/reduction during the electrochemical processes. Electropolymerization of conducting monomers was confirmed by increasing reduction-oxidation (redox) peaks $(\sim 1.0 \mathrm{~V})$ of poly $(\mathrm{G} 03 \mathrm{~T}-\mathrm{COOH})$ while the potential was swept from 0 to $1100 \mathrm{mV}$ from cycle 1 to 10 .

\section{Preparation of BPA-imprinted CD-PLL gel layers}

The BPA-imprinted CD-PLL gel layers were prepared using the method shown in Scheme 2. First, EDC (1.0 g, $5.22 \mathrm{mmol})$ and NHS $(1.0 \mathrm{~g}, 8.69 \mathrm{mmol})$ were dissolved in $20 \mathrm{ml}$ of ultra-pure water. Then, to activate carboxy groups on the surface of poly $(\mathrm{G} 03 \mathrm{~T}-\mathrm{COOH})$ films, the poly $(\mathrm{G} 03 \mathrm{~T}-\mathrm{COOH})$ films were immersed in the EDC/NHS solution for $1 \mathrm{~h}$ at room temperature. The films were rinsed with ultra-pure water and dried with a nitrogen flow. To form the CD-BPA-CD complexes, CD-PLL (100 mg) and BPA were dissolved in $10 \mathrm{ml}$ of ultra-pure water and stirred for $24 \mathrm{~h}$ at room temperature. By immersing the activated poly(G03T-COOH) films in the resultant CD-PLL solution for $2 \mathrm{~h}$, CD-PLL chains that form complexes with BPA were grafted onto the film surfaces, and $\mathrm{COOH}$ was activated with NHS. Then, the resultant CD-PLL gel layers were cross-linked via immersion in water that contained ethylene glycol diglycidyl ether $(10 \mathrm{wt} \%)$ for $24 \mathrm{~h}$ at $35^{\circ} \mathrm{C}$ in the presence of template BPA. BPA-imprinted CD-PLL gel layers were obtained by extracting the template BPA from the films using a water/acetone (70/30) mixture. Furthermore, non-imprinted CD-PLL gel layers were prepared by cross-linking CD-PLL gel layers in a similar manner without the template BPA.

To prepare a reference sensor chip, CD was directly immobilized on a sensor chip using a standard amino coupling method without using poly (G03T-COOH) and CD-PLL. First, to introduce carboxy groups onto a QCM sensor chip, the chip was immersed in ethanol that contained containing $5 \mathrm{mM}$ 3, 3'-dithiopropionic acid. Then, the sensor chip was washed with ethanol. Carboxy groups, which were introduced on the sensor chip, were activated with EDC $(1.0 \mathrm{~g})$ and NHS $(1.0 \mathrm{~g})$ in $20 \mathrm{ml}$ of ultra-pure water for $1 \mathrm{~h}$. After washing with water, the surface-activated sensor chip was immersed in 
$10 \mathrm{mg} \mathrm{ml}^{-1}$ of water that contained $\mathrm{NH}_{2}-\mathrm{CD}$ to obtain the directly CD-immobilized sensor chip.

\section{Characterizations of gel layers on QCM sensor chips}

The BPA-imprinted and non-imprinted CD-PLL gel layers were characterized using FT-IR-ATR, AFM and ellipsometry. FT-IR-RAS measurements were performed using a Spectrum 100 spectrometer (Perkin Elmer, Waltham, MA, USA) equipped with Refractor 2 (Harrick Scientific Inc., Pleasantville, NY, USA) with a single Brewster's angle silicon polarizer plate (grazing angle, $75^{\circ}$ ) and a liquid nitrogen-cooled MCT (Mercury-Cadmium-Telluride) detector (Perkin Elmer, Waltham, MA, USA). The spectra were referenced to a bare gold chip spectrum. All FT-IR-RAS measurements were performed at room temperature under dried air. In addition, the surface structure of BPAimprinted CD-PLL gel layers was observed with AFM in the tapping mode using SPI3800/SPA400 (SII nanotechnology, Tokyo, Japan) by taking 256 points in a $5000 \times 5000-\mathrm{nm}$ area with a $0.1 \mathrm{~Hz}$ scan rate at room temperature. The thickness of electropolymerized films on QCM sensor chips was determined using an M-2000 ellipsometer (JA Woollam Co., Lincoln, NE, USA) equipped with a laser with $250-1000 \mathrm{~nm}$ wavelengths (at $70^{\circ}, 75^{\circ}$ and $80^{\circ}$ angle of incidence).

\section{QCM measurements}

The adsorption of BPA, bisphenol E (BPE, 4,4'-ethylidenebisphenol) and bisphenol F (BPF, 4,4'-methylenebisphenol) into the BPA-imprinted and nonimprinted CD-PLL gel layers was evaluated with in situ QCM-D (model E1, Q-sense, Meiwa Fosis Co., Ltd, Tokyo, Japan). The changes in frequency and dissipation were monitored during the ultra-pure water flow. After the baseline was established, an aqueous solution of $400 \mu \mathrm{M}$ BPA, BPE or BPF was flown over the QCM sensors at a flow rate of $50 \mu \mathrm{min}^{-1}$ at $25^{\circ} \mathrm{C}$ for $80 \mathrm{~min}$. The frequency of the third overtone (F3) and dissipation (D3) was recorded. The subsequent changes in frequency were used to calculate the mass change due to BPA adsorption onto the film using the Sauerbrey equation. ${ }^{48}$

\section{RESULTS AND DISCUSSION}

\section{Characterizations of poly(G03T-COOH) films}

Cyclic voltammogram of $10 \mathrm{~mm}$ G03T-COOH on QCM sensor chips in acetonitrile with $0.1 \mathrm{M}$ tetrabutylammonium hexafluorophosphate is shown in Figure 1a. In the first cycle, the onset of the first anodic peak assigned as the oxidation of terthiophene pendant groups was observed at $\mathrm{E}_{\mathrm{pa}} 1.05 \mathrm{~V}$ versus $\mathrm{Ag} / \mathrm{AgCl}$ as a reference electrode. A cathodic peak was observed at $0.75 \mathrm{~V}$ versus $\mathrm{Ag} / \mathrm{AgCl}$. The oxidation and reduction peak currents increased gradually as the number of cyclic scans changed, which suggested a stepwise polyterthiophene film growth. Furthermore, the oxidation peak potential shifts in anodic direction and the reduction peak potential shifts in cathodic direction were observed on subsequent CV cycles.

The combination of CV with QCM allows us to monitor in situ the polymerization of G03T-COOH on QCM sensor chips through changes in the resonant frequency of the quartz crystal electrode. The negative value of the frequency change $(\Delta \mathrm{F})$ in Figure $1 \mathrm{~b}$ denotes that G03T-COOH was adsorbed onto the QCM crystal by electropolymerization and that the thickness of the formed film increased with $\mathrm{CV}$ cycles. QCM measurements revealed that $\Delta \mathrm{F}$ and the energy dissipation factor $(\Delta \mathrm{D})$ were shifted during the oxidation and reduction process. From both the QCM microgram and the $\mathrm{CV}$ diagram, we concluded that G03T-COOH was deposited to form a poly $(\mathrm{G} 03 \mathrm{~T}-\mathrm{COOH})$ film on a gold sensor chip. The thickness of the poly (G03T-COOH) film formed on the QCM sensor chip via electropolymerization was measured using ellipsometry. The poly (G03T-COOH) film prepared using CV (40 cycles) had an average thickness of $14.1 \mathrm{~nm}$. This means that a single CV scan resulted in the formation of a poly (G03T-COOH) film with an average thickness of $0.35 \mathrm{~nm}$, which is equal to the theoretical molecular thickness of
G03T-COOH $(\sim 0.39 \mathrm{~nm})$. Considering the strong interactions between sulfur moieties of the polyterthiophene backbone and the gold substrate, the closely related theoretical and experimental values suggest that a monolayer of poly $(\mathrm{G} 03 \mathrm{~T}-\mathrm{COOH})$ deposits on the gold substrate after each CV scan. Therefore, electropolymerization of G03T-COOH using CV enables us to form poly $(\mathrm{G} 03 \mathrm{~T}-\mathrm{COOH})$ films with a controlled thickness and to easily introduce functional carboxy groups onto QCM sensor chips. The modified QCM sensor chips with carboxy groups are very useful in fabricating highly sensitive QCM sensor chips with various ligands for a target molecule.

\section{Characterization of BPA-imprinted CD-PLL gel layers}

In this study, BPA was chosen as a target molecule because it has high possibility of exhibiting endocrine disrupting chemical behavior. The QCM technique is a powerful tool for detecting minute amounts of target biomolecules such as DNAs and proteins. However, QCM has a disadvantage in detecting small molecules with a very low molecular weight. Our strategy for detecting a small molecule, such as BPA, is to form molecularly imprinted gel layers on QCM sensor chips. In this study, CD-PLL was used to form BPA-imprinted gel layers on QCM sensor chips because amino groups of PLL can easily react with carboxy groups of poly(G03T-COOH) films on QCM sensor chips. After the complex formation between CD-PLL and BPA, CD-PLL was grafted onto the poly(G03T-COOH) films of QCM sensor chips.
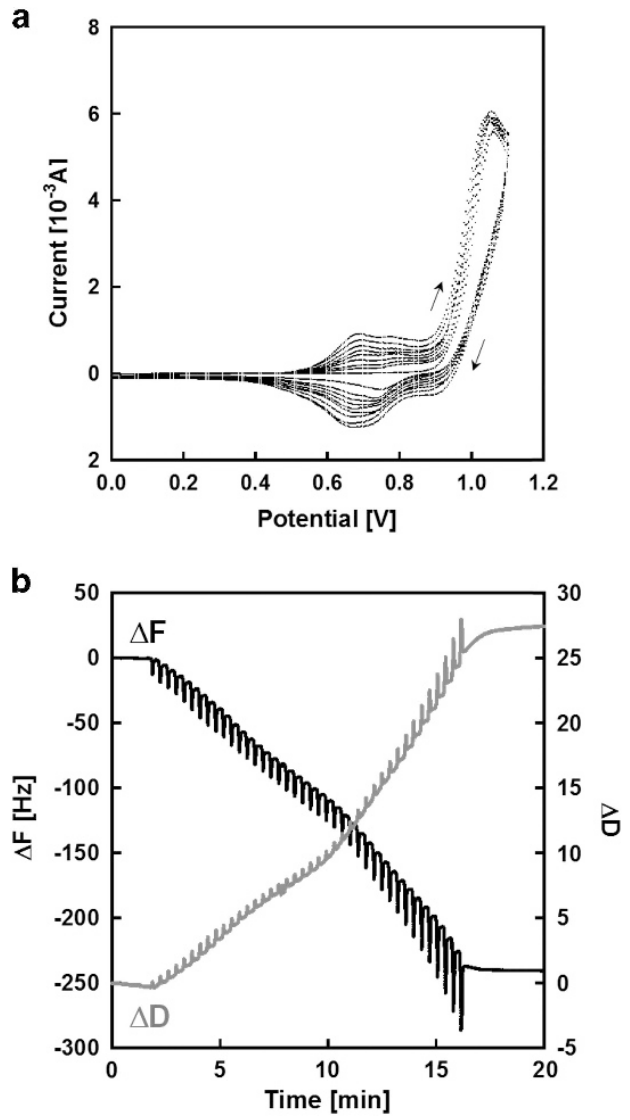

Figure 1 Cyclic voltammogram (a) and QCM response (b) of G03T-COOH on a QCM sensor chip in acetonitrile with $0.1 \mathrm{~m}$ tetrabutylammonium hexafluorophosphate. The concentration of $\mathrm{GO3T}-\mathrm{COOH}$ in acetonitrile was $10 \mathrm{~mm}$. Electropolymerization of $\mathrm{GO3T}-\mathrm{COOH}$ was performed by sweeping the potential from 0 to $1100 \mathrm{mV}$ vs the $\mathrm{Ag} / \mathrm{AgCl}$ reference electrode. A full color version of this figure is available at Polymer Journal online. 
Then, the BPA-imprinted CD-PLL gel layers were obtained by extracting template BPA from the CD-PLL gel layers. To extract BPA from the layers, the resulting sensor chips were immersed in a water/acetone mixture with an acetone concentration of $30 \mathrm{vol} \%$. The resultant BPA-imprinted CD-PLL gel layers were characterized using static water contact angle measurements, FT-IR-RAS spectroscopy, AFM and ellipsometry.

The water contact angles on a bare Au QCM sensor chip and a poly (G03T-COOH) film were found to be $46.3^{\circ}$ and $69.1^{\circ}$, respectively. After the formation of a BPA-imprinted CD-PLL gel layer on a poly (G03T-COOH) film on a QCM sensor chip, the water contact angle decreased from $69.1^{\circ}$ to $35.8^{\circ}$. The decrease in water contact angle implies that a hydrophilic CD-PLL gel layer was formed on a less hydrophilic poly $(\mathrm{G} 03 \mathrm{~T}-\mathrm{COOH})$ film by the reaction of CD-PLL with poly $(\mathrm{G} 03 \mathrm{~T}-\mathrm{COOH})$ in molecular imprinting. Figure 2 shows the FT-IR-RAS spectra of the poly $(\mathrm{G} 03 \mathrm{~T}-\mathrm{COOH})$ film and BPAimprinted CD-PLL gel layer. The poly(G03T-COOH) film FT-IR spectrum displays a peak at $\sim 1200 \mathrm{~cm}^{-1}$, which is assigned to the C-C stretching vibration, and peaks at $1456 \mathrm{~cm}^{-1}$ and $1510 \mathrm{~cm}^{-1}$, which are assigned to the $\mathrm{C}=\mathrm{C}$ stretching vibration bands of thiophene rings. Another strong peak at $1715 \mathrm{~cm}^{-1}$ was assigned to the carbonyl stretch vibration. In the spectrum of BPA-imprinted CD-PLL gel layer, there were strong absorption bands at $1540 \mathrm{~cm}^{-1}$ and $1650 \mathrm{~cm}^{-1}$ that corresponded to amide bonds of PLL. In

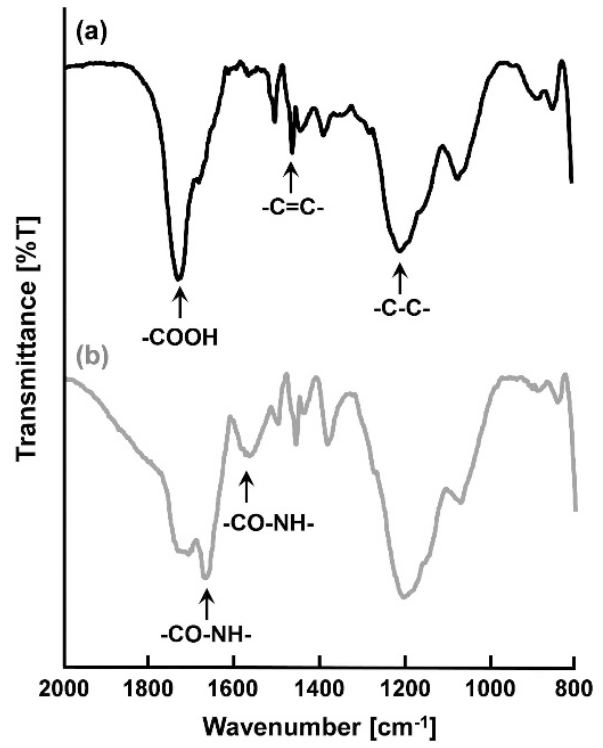

Figure 2 FT-IR-RAS spectra of a poly(G03T-COOH) film (a) and a BPA-imprinted CD-PLL gel layer (b). A full color version of this figure is available at Polymer Journal online.
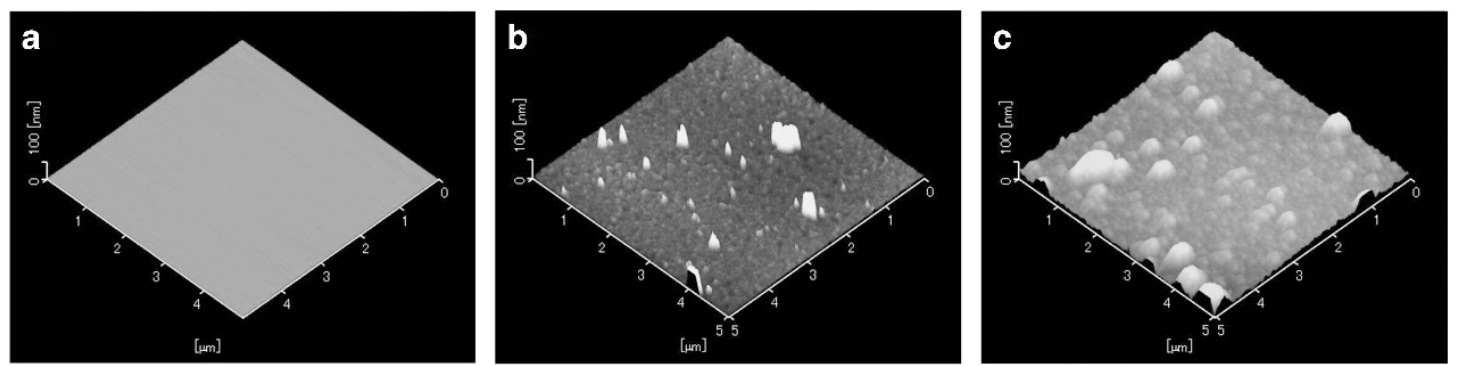

Figure 3 AFM images of a bare QCM sensor chip (a), a poly(G03T-COOH) film (b) and a BPA-imprinted CD-PLL gel layer (c). A full color version of this figure is available at Polymer Journal online. addition, the surface structure of BPA-imprinted CD-PLL gel layer was observed with AFM in the tapping mode at room temperature. Figure 3 shows AFM images of the bare QCM sensor chip, the poly $(\mathrm{G} 03 \mathrm{~T}-\mathrm{COOH})$ film and the BPA-imprinted CD-PLL gel layer. The AFM observation revealed that the poly $(\mathrm{G} 03 \mathrm{~T}-\mathrm{COOH})$ film and the BPA-imprinted CD-PLL gel layer with a large surface area were formed on the QCM sensor chip that was functionalized using electropolymerization of terthiophene. Ellipsometry revealed that the thicknesses of BPA-imprinted CD-PLL gel layer and non-imprinted CD-PLL gel layer on the QCM sensor chip were 15.7 and $19.5 \mathrm{~nm}$, respectively. The fact that the thickness of BPA-imprinted CD-PLL gel layer was approximately the same as that of non-imprinted CD-PLL gel layer implies that the same amount of CD-PLL was immobilized on the QCM sensor chips. Thus, we can design the BPA-imprinted CD-PLL gel layers in which CD ligands for the target BPA were arranged at optimal positions to form CD-BPA-CD complexes via molecular imprinting. The next section focuses on the BPA adsorption behavior of BPA-imprinted CD-PLL gel layers on QCM sensor chips.

$\mathrm{BPA}$ recognition behavior of $\mathrm{BPA}$-imprinted CD-PLL gel layers QCM is a powerful tool for monitoring molecular recognition events such as binding of lectins with carbohydrate and formation of DNA duplexes. In QCM techniques, molecular recognition events are monitored by weight changes that are induced by the adsorption of target biomolecules onto QCM sensor chips. Therefore, QCM has disadvantages in detecting a small molecule with a low molecular weight such as BPA. The BPA-imprinted CD-PLL gel layers on QCM sensor chips are expected to have many capacities for BPA adsorption because molecular recognition sites for a target BPA are effectively formed within PLL hydrogel networks by molecular imprinting using CDs as ligands. Therefore, BPA-imprinted CD-PLL gel layers on QCM sensor chips have a high possibility in detecting BPA even though BPA has a low molecular weight of $228 \mathrm{~g} \mathrm{~mol}^{-1}$. In this study, the adsorption behavior of BPA into BPA-imprinted CD-PLL gel layers was evaluated using in situ QCM-D measurements.

Figure 4 demonstrates QCM micrograms of a BPA-imprinted CD-PLL gel layer chip, a non-imprinted CD-PLL gel layer chip, a directly CD-immobilized chip and a bare chip in water that contained target BPA. The QCM measurements revealed that $\triangle \mathrm{F}$ of the BPA-imprinted CD-PLL gel layer chip decreased more than that of the non-imprinted CD-PLL gel layer chip that was prepared without a template BPA and the directly CD-immobilized chip. A larger decrease in $\triangle \mathrm{F}$ of the BPA-imprinted CD-PLL gel layer chip suggests that BPA-recognition sites were created via molecular imprinting using CD ligands. The $\triangle \mathrm{F}$ decrease of BPA-imprinted CD-PLL gel layer chip and non-imprinted CD-PLL gel layer chip corresponds to a mass gain of $205.8 \mathrm{ng} \mathrm{cm}^{-2}$ and $30.8 \mathrm{ng} \mathrm{cm}^{-2}$, respectively. This is attributed to the fact that molecular imprinting enabled CD ligands to be arranged at 
optimal positions for binding the target BPA even though our molecular imprinting used minute amounts of cross-linkers to form the gel layer (Figure 5). Furthermore, the sensitivity of QCM sensor chips may be improved by the large surface area of poly $(\mathrm{G} 03 \mathrm{~T}-\mathrm{COOH})$ films prepared via electropolymerization of terthiophene.

The sensitivity of BPA-imprinted CD-PLL gel layer on a QCM sensor chip was investigated by measuring $\triangle \mathrm{F}$ as a function of BPA concentration (Figure 6). In an aqueous solution with a BPA concentration of $<400 \mu \mathrm{M}, \Delta \mathrm{F}$ of the BPA-imprinted CD-PLL gel layer chip gradually increased with increasing BPA concentration in water. A calibration plot showing linearity between the BPA concentration and $\Delta \mathrm{F}$ in the concentration range between 0 and $400 \mu \mathrm{M}$ was obtained using the Pearson's correlation $R^{2}$ value of 0.9782 . The limit of detection (equal to $(3 \rho / m)$ ) and the limit of quantification (equal to $(10 \rho / m))$ in quantitative determination of BPA are 24.499 and $81.664 \mu \mathrm{M}$, respectively, where $\rho$ is the standard deviation and $m$ is the calibration curve slope. These values suggest that the BPA-imprinted CD-PLL gel layer chip is applicable to a QCM sensor device for

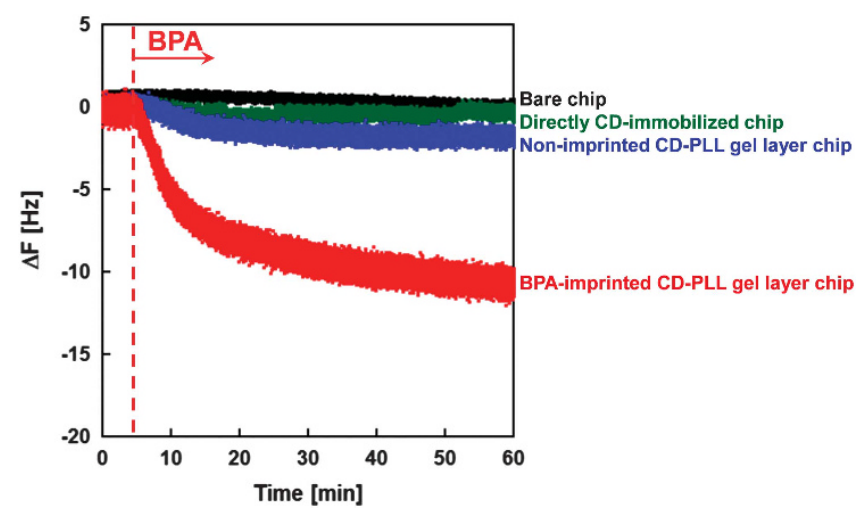

Figure 4 QCM responses of a BPA-imprinted CD-PLL gel layer chip, a non-imprinted CD-PLL gel layer chip, a directly CD-immobilized chip and a bare chip after the injection of an aqueous $400 \mu \mathrm{m}$ BPA solution. detecting BPA in water. Notably, the BPA-imprinted CD-PLL gel layer chip exhibited $\sim 7$ times greater $\Delta \mathrm{F}$ than the non-imprinted CD-PLL gel layer chip. Furthermore, in an aqueous solution with $<200 \mu \mathrm{M}$ $\mathrm{BPA}, \triangle \mathrm{F}$ of the non-imprinted CD-PLL gel layer chip became constant, which is different from the BPA-imprinted CD-PLL gel layer chip. This finding implies that the BPA-imprinted CD-PLL gel layer has a large molecular recognition capacity than the non-imprinted gel layer. The greater sensitivity of the BPAimprinted CD-PLL gel layer chip is attributed to the formation of BPA-recognition sites within its gel layer via molecular imprinting using the CD ligands. In addition, in our previous paper, we reported that BPA-imprinted bulk hydrogels that were prepared using poly (acrylamide) as the main chain exhibited a significant shrinkage in response to the target BPA. ${ }^{31}$ In BPA-imprinted bulk hydrogels, the BPA-recognition sites were formed via the optimal arrangement of ligand CDs. Therefore, the target BPA was preferentially absorbed into BPA-imprinted bulk hydrogels, which is similar to the BPA-imprinted CD-PLL gel layers on QCM sensor chips reported in this paper. However, BPA adsorption onto BPA-imprinted bulk hydrogels required a large amount of an aqueous BPA solution and several hours to attain equilibrium. In contrast, the BPA-imprinted CD-PLL gel layers on QCM sensor chips could rapidly detect the target BPA with a small amount of an aqueous BPA solution. Thus, the BPA-imprinted CD-PLL gel layers on QCM sensor chips provide useful platforms to fabricate molecular sensors that enable us to monitor BPA in aqueous solutions.

Molecular imprinting enables ligands to be arranged at optimal positions for a target molecule, followed by the formation of complementary cavities as molecular recognition sites. Standard molecular imprinting requires a large amount of cross-linkers to fix ligands at the optimal positions. However, because the BPA-imprinted CD-PLL gel layers on QCM sensor chips were weakly cross-linked using minute amounts of cross-linkers, they had flexible molecular recognition sites. We investigated the molecular recognition ability of BPA-imprinted CD-PLL gel layers by measuring $\triangle \mathrm{F}$ in an aqueous solution containing BPE and $\mathrm{F}$ (BPF) that have similar structures to BPA. QCM responses of the BPA-imprinted and non-imprinted

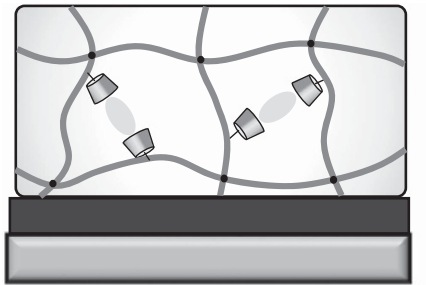

BPA-imprinted

CD-PLL gel layer

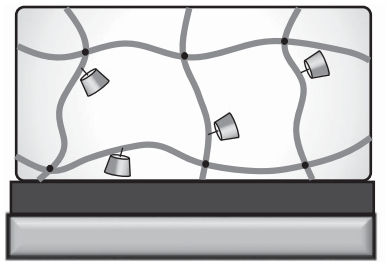

Non-imprinted CD-PLL gel layer
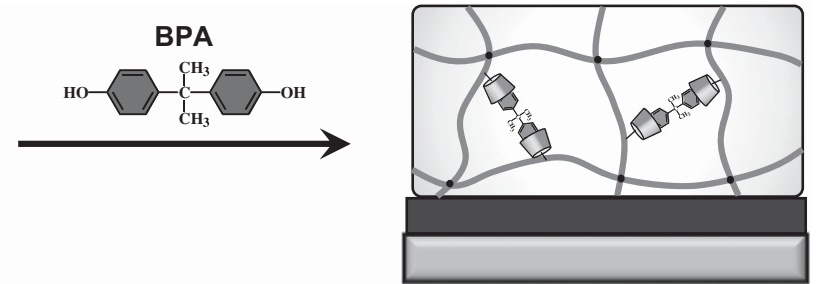

BPA recognition
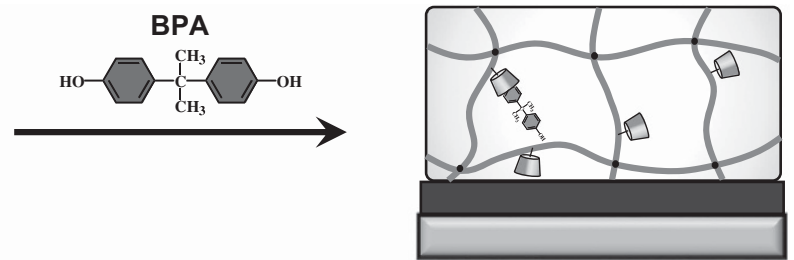

low recognition

Figure 5 Schematic illustration of BPA recognition of the BPA-imprinted CD-PLL gel layer and the non-imprinted CD-PLL gel layer. A full color version of this figure is available at Polymer Journal online. 
CD-PLL gel layer chips toward the injection of BPA, BPE and BPF are shown in Figure 7. The BPA-imprinted CD-PLL gel layer chip exhibited a much greater $\Delta \mathrm{F}$ than the non-imprinted CD-PLL gel layer chip in response to BPA, BPE and BPF. Because CD ligands in the BPA-imprinted CD-PLL gel layer were arranged at optimal positions for simultaneously binding two aromatic groups of BPA via molecular imprinting, the recognition sites that were formed using the template BPA also bonded BPE and BPF effectively. Thus, BPA and its derivatives were more efficiently adsorbed into the BPA-imprinted CD-PLL gel layer than into the non-imprinted CD-PLL gel layer. Notably, $\Delta \mathrm{F}$ of the BPA-imprinted CD-PLL gel layer chip for BPA was greater than that for BPE and BPF, even though the molecular structures of BPE and BPF are similar to that of BPA. We designed the BPA-imprinted CD-PLL gel layer chip via molecular imprinting using a minute amount of cross-linkers, which is differing from a standard molecular imprinting that requires a large

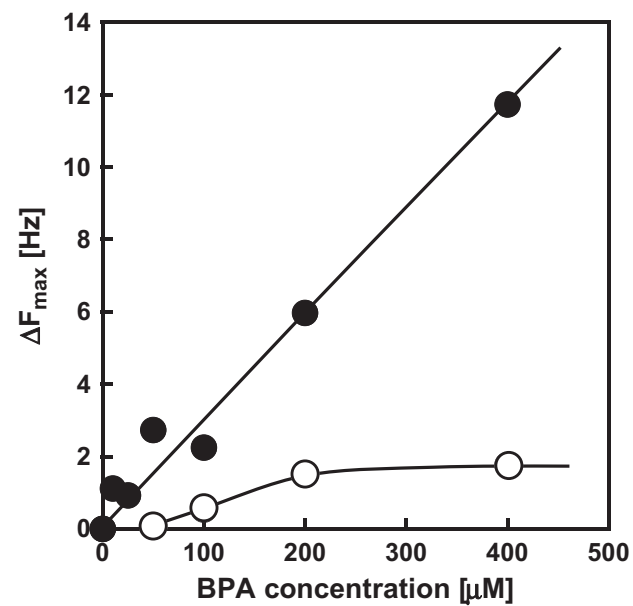

Figure 6 Relationship between the BPA concentration in water and QCM responses of the BPA-imprinted CD-PLL gel layer chip (-) and the nonimprinted CD-PLL gel layer chip ( $\circ$ ) after the injection of an aqueous BPA solution amount of cross-linkers. The fact that BPA was more efficiently adsorbed into the BPA-imprinted CD-PLL gel layer than BPE and BPF reveals that the recognition sites formed by molecular imprinting can recognize a small difference between $\mathrm{BPA}$ and $\mathrm{BPE} / \mathrm{BPF}$. This may be attributed to the molecular recognition ability of $\mathrm{CDs}$ and to the important role of amino groups of PLL main chains. The high recognition ability and multiple interactions in molecular imprinting enable the resulting gel layers to recognize target molecules selectively and sensitively despite weak cross-linking. Although further research is required to clarify the molecular recognition behavior of BPA-imprinted CD-PLL gel layers, the molecularly imprinted gel layers that are weakly cross-linked on QCM sensor chips are likely to become promising sensor chips for monitoring small amounts of BPA and its derivatives slightly in aqueous solutions. Molecular imprinting using PLL and minute amount of cross-linkers will contribute significantly to the fabrication of highly sensitive and selective QCM sensor systems.

\section{CONCLUSION}

This paper describes the preparation of molecularly imprinted polypeptide gel layers with molecular recognition sites on QCM sensor chips by combining electropolymerization with molecular imprinting. Electropolymerization of G03T-COOH was performed to form poly(G03T-COOH) films on QCM sensor chips. The process was monitored using in situ EQCM measurements. The BPA-imprinted CD-PLL gel layers with molecular recognition sites were prepared on the poly $(\mathrm{G} 03 \mathrm{~T}-\mathrm{COOH})$ films of QCM sensor chips via chemical cross-linking of PLL and molecular imprinting that used CDs as ligands for the template BPA. In QCM measurements, the BPA-imprinted CD-PLL gel layer chip showed a greater $\Delta \mathrm{F}$ in response to BPA than the non-imprinted CD-PLL gel layer chip and the directly CD-immobilized chip. This is attributed to a large surface area produced by electropolymerization and the arrangement of $\mathrm{CD}$ ligands at optimal positions for forming CD-BPA-CD complexes via molecular imprinting. The QCM microgram demonstrated a very good linear relationship between the BPA concentration and $\Delta \mathrm{F}$ in the concentration range between 0 and $400 \mu \mathrm{M}$. The BPA-imprinted

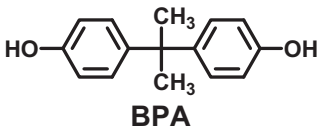

a

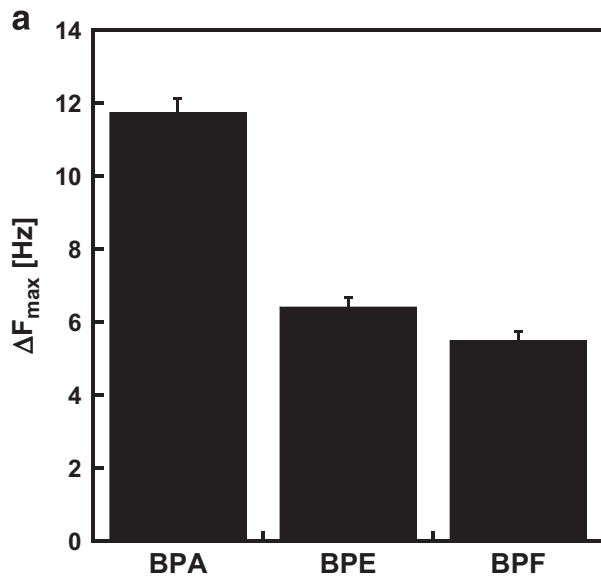

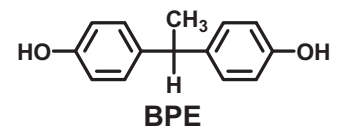

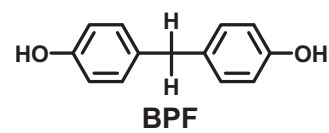

b

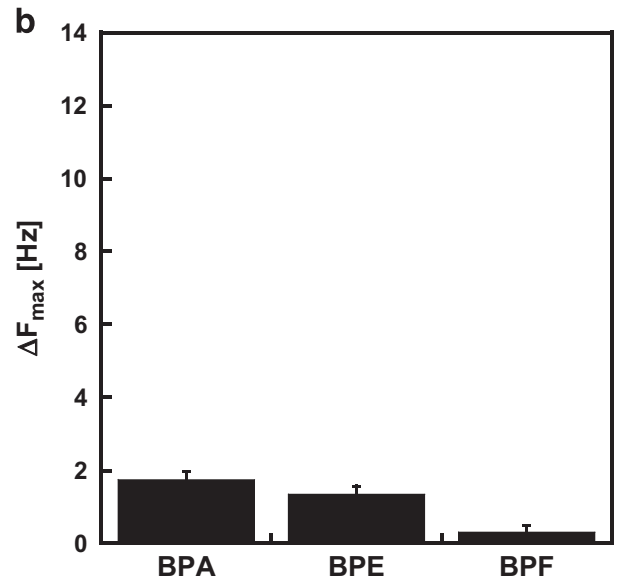

Figure 7 QCM responses of the BPA-imprinted CD-PLL gel layer chip (a) and the non-imprinted CD-PLL gel layer chip (b) after the injection of $400 \mu \mathrm{m}$ BPA, $\mathrm{BPE}$ and BPF aqueous solutions. 
CD-PLL gel layers that are weakly cross-linked on QCM sensor chips are likely to become promising sensor chips for monitoring BPA and its derivatives with small amounts in aqueous solutions.

\section{CONFLICT OF INTEREST}

The authors declare no conflict of interest.

\section{ACKNOWLEDGEMENTS}

This work was supported in part by the Grant-in-Aid for Scientific Research on Innovative Areas of 'New Polymeric Materials Based on Element-Blocks' (No. 15H00768) from The Ministry of Education, Culture, Sports, Science and Technology of Japan, by the Grant-in-Aid for Scientific Research (B; No. 15H03026) from the Japan Society for the Promotion of Science (JSPS), and by the grants from the National Science Foundation (NSF STC-0423914 and NSF CMMI 1333651).

1 Kavlock, R. J., Daston, G. P., DeRosa, C., Fenner-Crisp, P., Gray, L. E., Kaattari, S., Lucier, G., Luster, M., Mac, M. J., Maczka, C., Miller, R., Moore, J., Rolland, R., Scott, G., Sheehan, D. M., Sinks, T. \& Tilson, H. A. Research needs for the risk assessment of health and environmental effects of endocrine disruptors: a report of the U.S. EPA-sponsored workshop. Environ. Health Perspect. 104, 715-740 (1996).

2 Kim, H. S., Han, S. Y., Yoo, S. D., Lee, B. M. \& Park, K. L. Potential estrogenic effects of bisphenol-A estimated by in vitro and in vivo combination assays. J. Toxicol. Sci. 26 111-118 (2001)

3 Li, W., Seifert, M., Xu, Y. \& Hock, B. Comparative study of estrogenic potencies of estradiol, tamoxifen, bisphenol-A and resveratrol with two in vitro bioassays. Environ. Int. 30, 329-335 (2004).

4 Suzuki, A., Sugihara, A., Uchida, K., Sato, T., Ohta, Y., Kats, Y., Watanabe, H. \& Iguchi, T. Developmental effects of perinatal exposure to bisphenol-A and diethylstilbestrol on reproductive organs in female mice. Reprod. Toxicol. 16, 107-116 (2002).

5 Malitesta, C., Losito, I. \& Zambonin, P. G. Molecularly imprinted electrosynthesized polymers: new materials for biomimetic sensors. Anal. Chem. 71, 1366-1370 (1999).

6 Dickert, F. L., Hayden, O. \& Halikias, K. P. Synthetic receptors as sensor coatings for molecules and living cells. Analyst 126, 766-771 (2001).

7 Lin, J. M. \& Yamada, M. Chemiluminescent flow-through sensor for 1,10-phenanthroline based on the combination of molecular imprinting and chemiluminescence. Analyst 126, 810-815 (2001).

8 Apodaca, D., Pernites, R., Ponnapati, R., Del Mundo, F. \& Advincula, R. Electropolymerized molecularly imprinted polymer films of a bis-terthiophene dendron: folic acid quartz crystal microbalance sensing. ACS Appl. Mater. Interfaces 3, 191-203 (2011)

9 Pernites, R., Ponnapati, R. \& Advincula, R. Surface plasmon resonance (SPR) detection of theophylline via electropolymerized molecularly imprinted polythiophenes. Macromolecules 43, 9724-9735 (2010).

10 Pernites, R., Ponnapati, R., Felipe, J. \& Advincula, R. Electropolymerization molecularly imprinted polymer (E-MIP) SPR sensing of drug molecules: pre-polymerization complexed terthiophene and carbazole electroactive monomers. Biosens. Bioelectron. 26, 2766-2771 (2011).

11 Mosbach, K. Molecular imprinting. Trends Biochem. Sci. 19, 9-14 (1994).

12 Shea, K. J. Molecular imprinting of synthetic network polymers: the de novo synthesis of macromolecular binding and catalytic sites. Trends Polym. Sci. 2, 166-184 (1994).

13 Wulff, G. Molecular imprinting in cross-linked materials with the aid of molecular templates- a way towards artificial antibodies. Angew. Chem. Int. Ed. 34 1812-1832 (1995).

14 Byrnea, M. E., Parka, K. \& Peppas, N. A. Molecular imprinting within hydrogel. Adv. Drug Delivery Rev. 54, 149-161 (2002).

15 Asanuma, H., Hishiya, T. \& Komiyama, M. Tailor-made receptors by molecular imprinting. Adv. Mater. 12, 1019-1030 (2000).

16 Hishiya, T., Shibata, M., Kakazu, M., Asanuma, H. \& Komiyama, M. Molecularly imprinted cyclodextrins as selective receptors for steroids. Macromolecules 32 , 2265-2269 (1999)

17 Tanaka, T., Fillmore, D., Sun, S.-T., Nishio, I., Swislow, G. \& Shah, A. Phase transitions in ionic gels. Phys. Rev. Lett. 45, 1636-1644 (1980).

18 Annaka, M. \& Tanaka, T. Multiple phases of polymer gels. Nature 335, 430-432 (1992).

19 Amiya, T., Horikawa, Y., Hirose, Y., Li, Y. \& Tanaka, T. Reentrant phase transition of $\mathrm{N}$-isopropylacrylamide gels in mixed solvents. J. Chem. Phys. 86, 2375-2379 (1987).
20 Chen, G. \& Hoffman, A. S. Graft copolymers that exhibit temperature-induced phase transitions over a wide range of $\mathrm{pH}$. Nature 373, 49-52 (1995).

21 Yoshida, R., Uchida, K., Kaneko, T., Sakai, K., Kikuchi, A., Sakurai, Y. \& Okano, T. Comb-type grafted hydrogels with rapid deswelling response to temperature changes. Nature 374, 240-242 (1995)

22 Tanaka, T. Gels. Sci. Am. 244, 124-136 (1981).

23 Dusek, K. Responsive Gels: Volume Transitions I. Adv. Polym. Sci. 109 (Springer, Berlin, Germany, 1993)

24 Dusek, K. Responsive Gels: Volume Transitions II. Adv. Polym. Sci. 110 (Springer, Berlin, Germany, 1993).

25 Okano, T. Biorelated Polymers and Gels (Academic, Boston, MA, USA, 1998).

26 Hoffman, A.S. "Intelligent" polymers in medicine and biotechnology. Macromol. Symp. 98, 645-664 (1995)

27 Miyata, T. Preparation of smart soft materials using molecular complexes. Polym. J. 42, 277-289 (2010)

28 Miyata, T., Asami, N. \& Uragami, T. Structural design of stimuli-responsive bioconjugated hydrogels that respond to a target antigen. J. Polym. Sci. Part B: Polym. Phys. 47, 2144-2157 (2009).

29 Miyata, T., Jige, M., Nakaminami, T. \& Uragami, T. Tumor marker-responsive behavior of gels prepared by biomolecular imprinting. Proc. Natl Acad. Sci. USA 103, 1190-1193 (2006)

30 Miyata, T., Asami, N. \& Uragami, T. A reversibly antigen-responsive hydrogel. Nature 399, 766-769 (1999)

31 Kawamura, A., Kiguchi, T., Nishihata, T., Uragami, T. \& Miyata, T. Target moleculeresponsive hydrogels designed via molecular imprinting using bisphenol $\mathrm{A}$ as a template. Chem. Commun. 50, 11101-11103 (2014).

32 Matsumoto, K., Kawamura, A. \& Miyata, T. Structural transition of pH-responsive poly(L-lysine) hydrogel prepared via chemical cross-linking. Chem. Lett. 44, 1284-1286 (2015).

33 Advincula, R., Brittain, B., Ruhe, J. \& Caster, K. Polymer Brushes: Synthesis Characterizations, Applications (Wiley- VCH, Weinheim, Germany, 2004).

34 Duran, H., Ogura, K., Nakao, K., Vianna, S., Usui, H., Advincula, R. \& Knoll, W. High-vacuum vapor deposition and in situ monitoring of $\mathrm{N}$-carboxy anhydride benzyl glutamate polymerization. Langmuir 25, 10711-10718 (2009).

35 Wang, X., Kim, Y., Drew, C., Ku, B., Kumar, J. \& Samuelson, L. Electrostatic assembly of conjugated polymer thin layers on electrospun nanofibrous membranes for biosensors. Nano Lett. 4, 331-334 (2004).

36 Pernites, R., Venkata, S., Tiu, B., Yago, A. \& Advincula, R. Nanostructured, molecularly imprinted, and template-patterned polythiophenes for chiral sensing and differentiation. Small 8, 1669-1674 (2012).

37 Park, J., Pernites, R.,. Estillore, N., Hyakutake, T., Ponnapati, R., Tiu, B., Nishide, H. \& Advincula, R. Capsulation of carbon nanotubes on top of colloidally templated and electropolymerized polythiophene arrays. Chem. Commun. 47, 8871-8873 (2011).

38 Tong, H., Wang, L., Jing, X. \& Wang, F. "Turn-on" conjugated polymer fluorescent chemosensor for fluoride ion. Macromolecules 36, 2584-2586 (2003).

39 McCullough, R., Ewbank, P. \& Loewe, R. Self-assembly and disassembly of regioregular, water soluble polythiophenes: chemoselective ionchromatic sensing in water. J. Am. Chem. Soc. 119, 633-634 (1997).

40 Tiu, B., Pernites, R., Foster, E. \& Advincula, R. Conducting polymer-gold co-patterned surfaces via nanosphere lithography. J. Colloid Interface Sci. 459, 86-96 (2015).

41 Evans-Kennedy, U., Clohessy, J. \& Cunnane, V. J. Spectroelectrochemical study of $2,2^{\prime}: 5^{\prime}, 2^{\prime \prime}$-terthiophene polymerization at a liquid/liquid interface controlled by potential-determining ions. Macromolecules 37, 3630-3634 (2004).

42 Zotti, G., Marin, R. \& Gallazzi, M. Electrochemical polymerization of mixed alkyl-alkoxybithiophenes and -terthiophenes. Substitution-driven polymerization from thiophene hexamers to long-chain polymers. Chem. Mater. 9, 2945-2950 (1997).

43 Taranekar, P., Fulghum, T., Baba, A., Patton, D. \& Advincula, R. Quantitative electrochemical and electrochromic behavior of terthiophene and carbazole containing conjugated polymer network film precursors: EC-QCM and EC-SPR. Langmuir 23, 908-917 (2007)

44 Byun, H. S. \& Bittman, R. Hydrophilic cholesterol-binding molecular imprinted polymers. Tetrahedron Lett. 42, 1839-1841 (2001).

45 Prabaharan, M. \& Mano, J. F. Hydroxypropyl chitosan bearing $\beta$-cyclodextrin cavities: synthesis and slow release of its inclusion complex with a model hydrophobic Drug. Macromol. Biosci. 5, 965-973 (2005).

46 Girek, T. \& Ciesielski, W. Polymerization of $\beta$-cyclodextrin with succinic anhydride and thermogravimetric study of the polymers. J. Incl. Phenom. Macrocycl. Chem. 69, 439-444 (2011).

47 Adrian, F., Budtova, T., Tarabukina, E., Pinteala, M., Mariana, S., Peptu, C., Harabagiu, V. \& Simionescu, B. C. Inclusion complexes of $\gamma$-cyclodextrin and carboxyl-modified $\gamma$-cyclodextrin with C60: synthesis, characterization and controlled release application via microgels. J. Incl. Phenom. Macrocycl. Chem. 64, 83-94 (2009)

48 Sauerbrey, G. Z. Verwendung von schwingquarzen zur wägung dünner schichten und zur mikrowägung. Phys. A Hadrons Nucl. 155, 206-222 (1959). 